\title{
Study on Wireless Sensor Network Services Model in Basis of IPv6
}

\author{
Jianlan Liu \\ College of Mathematics and Computer Science \\ Jiangxi Science and Technology Normal University \\ Nanchang, China \\ E-mail: ljlan0821@163.com
}

\begin{abstract}
Wireless sensor network is an intelligent sensor network system collected with data acquisition, data processing and communication in one, IPv6, as the core protocol of next-generation internet, has ample of address space, address auto-configuration and mandatory safety measures, and provides the feasibility for large-scale application of wireless sensor networks; by studying the technology that access wireless sensor network to internet with IPv6 as the core, people design and take use of experimental systems to verify services model and optimize multi-user service requests, while wireless sensor networks reduces the number of internal inquiries, lowers energy consumption and prolongs the network life cycle which allows users to easily access the sensor network resources via internet.
\end{abstract}

Keywords-Wireless Sensor Network; IPv6; Gateway; Routing Protocol; Service Request

\section{INTRODUCTION}

Wireless sensor networks have multiple functions like data acquisition, data processing, wireless communications in researches on knowledge highly integrated and multi-disciplinary, which also plays an important role in the military, medical, environmental monitoring, smart home, space exploration and other fields [1]; continuous development of wireless sensor networks and Internet technology has more and more requirements for sensor network to access Internet; and also the technical characteristics of IPv6 such as IP header format, a larger address space, hierarchical routing structure, stateless and state address configuration, multicast technology, etc. provide a good technical support for the development of wireless sensor networks, therefore, it is in line with the developmental direction of wireless sensor network technology to studying on accessing wireless sensor networks to Internet based on IPv6 protocol and making the two organically integrate and effectively communicate.

\section{WIRELESS SENSOR NET WORK SERVICES MODEL BASED ON IPV6}

In existing wireless sensor network reference model, this model adds a service management platform to provide a unified service interface for sensor networks to access into Internet so that users can access the relevant monitoring area and obtain monitoring data only in accordance with the mapping of IP (v6) address. The model also provides methods to optimize the multi-user service requests and effectively resolve the problem of excessive energy consumption due to a large number of repeat users and their frequent query on sensor network.

\section{A. Service Model Structure}

Existing TCP / IP Protocol cannot be used directly on a wireless sensor network which causes out that network users can not directly access the wire less sensor networks, so it cannot easily unify the port for wireless sensor networks access to internet unless add and rational design service model in the existing sensor network reference model [2].

Integrated on the services gateway, the service management platform is composed by physical layer, data transform layer (DTL), network layer (NL), service strategy layer (SSL) and service interface layer (SIL) which corresponding to wireless sensor network reference model layers: SIL use Socket port programming to realize information exchange with network users; SSL solve the optimization problems of the multi-user service requests; NL deal with issues as address mapping and routing protocol; DTL will transform the collected data into data format which can be understood by upper layer and also the users' service requests into inquiry packet that can be understood by sensor network, Fig. 1 shows a serving gateway protocol.

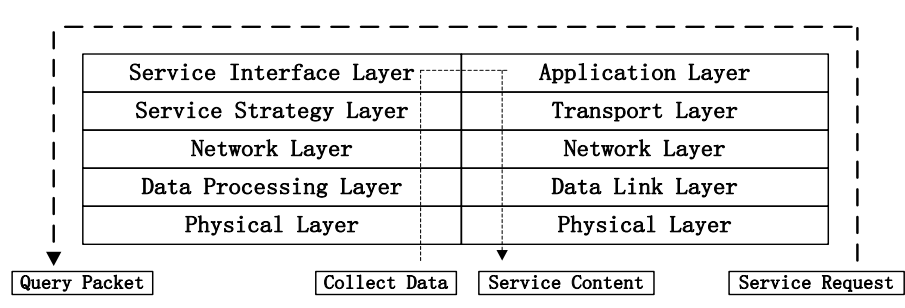

Figure 1. Serving Gateway Protocol

\section{B. Wireless Sensor Network Routing Protocol}

After receiving users' service request, the gateway parses correspondence with the sensor network monitoring area out from the 16-bit redundancy in IPv6 address, and then wireless sensor networks build the path to the destination node by using location-based routing protocol [3]. Common location routings have GTBRA, GAF, GEAR, and etc. among which GEAR is a kind of typical location-based routing protocol [4] usually used by wireless sensor networks. When users access wireless sensor networks, the service gateway 
parses the IPv6 address to corresponding monitoring area and then establish a path leading to the monitoring area according to GEAR, then the first node that receives information in this monitoring area will send a command of requesting data collection to other nodes within this network through flooding or iteration forwarding mechanism, and the other nodes will then transmit the collected data back to the first node after receiving the command, the trans mitted data at the same be integrated and at the first node and finally the information will be sent back to service gateway.

\section{Model Design}

\section{1) Packet format design}

The internal transfer packet format of wireless sensor network has two broad categories: one is data query group (DQG) and the other is data transfer group (DTG), the former one is to send query of collecting environmental information from service gateway to wireless sensor network and so the default source address is service gateway; while after the leaf nodes receive packet of DQG, driver sensors chip begins to collect the environmental information and then the leaf nodes collect the data to packet into DTG packet format and transfer the data to service gateway progressively through routing nodes, and so all DTG packets are sent to service gateway which consequently is the default destination address.

DQG packet format is 32 bits and for DTG is 64 bits, each of the bits showed below table I and table II:

T ABLE I. DQGPACKETS FORMAT

\begin{tabular}{|c|c|r|c|c|c|c|}
\hline Bit & $31: 30$ & $29: 24$ & $23: 8$ & $7: 2$ & 1 & 0 \\
\hline Function & Group Type & Retention & $\begin{array}{c}\text { Destination } \\
\text { Address }\end{array}$ & Retention & Temperature Flag Bits & Humidity Flag Bits \\
\hline
\end{tabular}

T ABLE II. DT GPACKETS FORMAT

\begin{tabular}{|c|c|c|c|c|c|c|c|}
\hline Bit & $63: 62$ & $61: 48$ & $47: 32$ & 31 & 30 & $29: 16$ & $15: 0$ \\
\hline Function & Group Type & Retention & $\begin{array}{c}\text { Node } \\
\text { Number }\end{array}$ & $\begin{array}{c}\text { Temperature } \\
\text { Flag Bits }\end{array}$ & $\begin{array}{c}\text { Humidity } \\
\text { Flag Bits }\end{array}$ & $\begin{array}{c}\text { Retention } \\
\text { Collection } \\
\text { Value }\end{array}$ \\
\hline
\end{tabular}

Group Type: 01 presents DQG packets, 10 presents DTG packets

Destination Address: Each sub-network mapping coordinates, has total 16 bits;

Temperature/ Hu midity Flag Bits: 1 request ambient temperature or humidity data, 0 indicates the contrary;

Node Number: Information collected leaf node number, with 16 bits in total;

Temperature/ Humidity Flag Bits: Set effection, 1 indicates temperature or humidity values are collected;

Collection Value: the actual data collected by nodes.

2) Communication Software Design

First of all, the deployment location of sensor nodes in model design is fixes and a global routing table shall be set up in each node which will accordingly choose proper path to correctly forward the received group and then design the table into a two-dimensional array to manage; secondly, the data packet is progressively transmitted through routing node in a wireless communication system, after powered, the nodes begin to call the initialization function to initialize work; finally, each routing node computes its located grid and simultaneously set the node to a state of monitoring and receiving wireless signal, once monitoring data transmitted, each of them shall start receiving function to analyze and process the received data, and then the state of it shall be set back to the one of monitoring and receiving wireless signal.

\section{3) Service Gateway Software Design}

First, the experimental model access to Internet based on IPv6 through the gateway, the access gateway is composed by an enhanced transmitting power CC2430 wireless sensor nodes and an operating system installed Redhat 9.0 x 86 host; the $\times 86$ host access to the internet through RJ-45 Ethernet port, the sensor node connects to the host via RS-232 serial interface, and the connecting way for sensor network and CC2430 sensor node is wireless.

\section{4) Service Interface Layer Design}

Through the use of socket programming and TCP link, user can implement an information exchange with wireless sensor network interface layer, the specific steps show:

\section{a) Create Socket}

The user call socket () system to create a socket with service interface, and domain the specified socket as PF_INET6 and the service type as SOCK_STREAM, and the protocol defaults to 0 :

int socket (int domain, int type, int protocol);

b) Name and Bind a Socket

To facilitate other processes via socket () system call created socket, the user firstly give the created socket a name and then call to bind () system to bind the socket; the special address socket structure in $\operatorname{IPv} 6$ is "struct sockaddr_in6":

struct sockaddrint

\{

u_long sin6_flowinfo; // * marked traffic * //

u_short sin $6 \_$port; //* port number $*$

short $\sin 6 \_$family; // * IPv6 address family * //

struct in6_addr sin6_addr; // * IPv6 in the 16-byte structure $* / /$

u_long sin6_scope_id; // *IPv6 address of the interface index range ID *//

;

int bind (int socket, const struct sockaddr $*$ address, size_t address_len);

c) Create Socket Queue

When the program is being processed, the port may have a new socket request, then the service gateway 
calls listen () program to create a socket queue and save a new socket to accept incoming requests and waiting to be processed.

int listen (int socket, int number);

d) Accept Connections

After the server socket is created and named, the user can call accept () program to set connections with the socket.

int accept (int socket, struct sockaddr * address, size_t $*$ address_len);

e) Parse IP Addresses

This is completed through calling accept () procedure, connecting users socket request, resolving the IP address of the user service request by service gateway and then call getsockname () function to return a specific IP address to the user.

int getsockname(int sock, struct sockaddr *name, socklen_t*namelen);

\section{f) Create a Child Process}

After the running of user socket requesting access, service gateway calls fork () program to create a child process and at the same time the father process returns to continuously monitor later received socket request by which multiple user terminal service requests can be processed simultaneously.

g) Close the Socket

After processing all the sockets, the gateway calls close () function to close the connection socket for the user and wireless sensor networks.

int close (int socket);

5) Service Strategy Layer Design

There is large and frequent check request from users to sensor network enter and queue in the socket queue of service strategy layer, so if with no corresponding control mechanism, the service strategy layer shall actively responds to every check requests that the energy of sensor network will be quickly depleted [5]; the optimization strategy sub-process of service strategy layer is to optimize and control the plenty service requests in order to avoid the sensor network energy consumption of too fast; the design of service optimization strategy sub-process needs database support, so that two memory table named WSN_DATA and WAIT_QUEUE are designed here in MySQL database in which the former one is used to store data collection while the second one to be as query pool for service requests storage.

\section{6) Data Transform Layer Design}

Data transform layer in service gateway is mainly to reasonably dispatch the users' servicer requests that are waiting in query pool for processing, so a background process is built in service gateway to periodically query service requests awaiting in the scheduling of
WATI_QUEUE table, and each of the service requests is queued in query pool according to waiting time in which the service request that with longest waiting time will be transformed into DQG query packet by the process who will at the same transform the DTG packet into collected data to store in WSN_DATA storage table.

\section{ANALYSIS OF EXPERIMENT AL RESULTS}

Applying the principles stated above, the experimental system is designed to verify the working performance of the service model accessing wireless sensor network to Internet based on IPv6, and the system consists of room service gateways and a small wireless sensornetworks composed of 15 sensornodes.

\section{A. Experimental Environment}

The whole experiment system is deployed indoors, and the 15 wireless sensor nodes are fixed at aisles laboratory in the same floor in advance, and with the method of wireless multi-hop communication, the nodes compose into a small wireless sensor network which access to Internet based on IPv6 through the service gateway set at the other side; the area of corresponding monitoring is $40 \mathrm{~m} \times 40 \mathrm{~m}$ which is divided into $4 \mathrm{sub}$ grids with area of $20 \mathrm{~m} \times 20 \mathrm{~m}$, and each of the sub grid maps one IPv6 address, then the mapped IP address will be noted in the IP address table that has been built in service gateway.

\section{B. Performance Measurement and Analysis}

This experiment conducts the system test in a virtual local area network, and one of the two x86 hosts is set a new user terminal which uses IPv6 address and port for requesting service to imitate actual behavior; the other one uses the Red Hat release 9-linux operating system and mysql database as its service gateway.

1) Single User Behavior Test

In the single-user behavior performance test experiment, one new user terminal is set and with random IPv 6 address and port, it separately launches 10 times, 20 times ...60 times checking service requests, and the wireless sensor networks is shut down during the test in order to ignore the effects of test results because of different time delay in actual acquisition of wireless sensor networks; assuming the minimum time interval $\triangle \mathrm{TIME}$ of multi-user service requests optimization strategy set is 60 seconds and under the condition of respectively closing and opening multiuser service requests optimization, the average response time for wireless sensor networks queries and service requests obtains corresponding responding results with the increase of the number of user queries. 


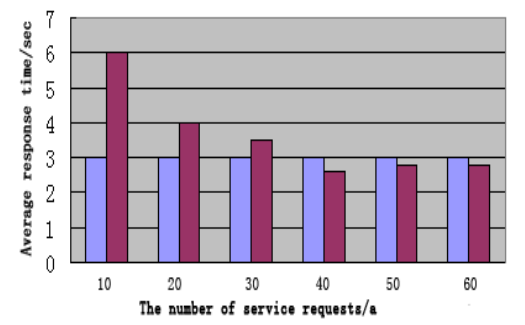

Figure 2. Average Respond Time

As shown in Fig. 2 and Fig. 3, ORDINARILY indicates the test result of multi-user service reques ts to optimize, and STRATEGY shows the result after starting the multi-user service requests optimization strategy. The experiment shows that when service request is less frequent, ORDINARILY takes the form of immediate dealing for service requests and so the average respond time is quick, but it is slower for STRATEGY for it has a periodically scheduling forwarding.

\section{2) Multi User Behavior Test}

In this test, 2 user terminals, 4 user terminals and 12 user terminals is set respectively for one time, and each time the terminals send simultaneously inquiry service request to the wireless sensor network with random IPv6 address and port number; the test tests out response of the service gateway to multi-user terminal services requests under the condition of closing and starting multi-user service requests optimization strategy.

It is obtained by analyzing the results that regardless of how many network users concurrently request service the average waiting time for each user are the same in ORDINARILY, because ORDINA RILY adopts positive response strategy that make active response every time when users query service and its number of queries to sensor network increases simultaneously with the increase of user; but for STRATEGY, the average waiting time will decrease with the increase of users and the query time to sensor network will almost keep unchanged when it comes up to 5 , it is because that service requests from multiple users are in the same monitoring area and STRATEGY do the integration process which can meet the needs of multi-user services by using less queries.

In summary of testing results above, multi-user service request optimization strategy can take optimizing control on plenty of frequent, repeated or region overlapping user service requests, which reduces unnecessary queries to internal wireless sensor networks, so as to greatly reduce the wireless sensor networks internal energy consumption and extend the life cycle of it.

\section{CONCLUSION}

By studying the technology of accessing wireless sensor network to Internet with IPv6 protocol as its core,

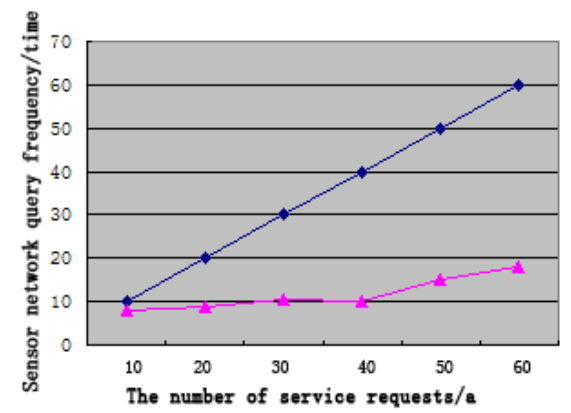

Figure 3. Sensor Network Query Times

it allows network users can easily access sensor network resources via Internet and use designed experimental system to verify the performance of the service model; the model can take optimization to multi-user service requests that reduces the query times within the network, lowers energy consumption and extend the network lifetime [6]; however, the experimental system is a demonstration system and it is processed at fixed indoor place with few nodes that have fixes location and simple tasks, it has only one service gateway to get access to Internet, while in large-scale wireless sensor network scenario, multiple service gateways shall be deployed. Consequently, it needs to be further extended and researched in the key technologies like interactive cooperation of multi-service gateways, the optimizing control on multi-service requests and so on.

\section{REFERENCES}

[1] Wen, Zhiping; Li, Xun; Li, Hongjun, Design and Implementation of Wireless Sensor Network IPv6 Access System [J], Computer Engineering, 2010.02.

[2] Wang, Xiaonan; Gao, Demin; Qian, Huanyan, IPv6 Address Auto-configuration Scheme in Wireless Sensor Network [J], Paper of Electronic Science University, 2011.05.

[3] Hu, Ting, Wireless Sensor Networks and IPv6 Networking Solutions Research [D], Beijing: Beijing Posts and Telecommunications University, 2010.01.

[4] Chen, Huaiyou, Virtual Reality Technology [M], Beijing: T singhua University Press, 2012.

[5] Sun, Jianzhi, Routing Algorithm for Opportunistic Networks [M], Beijing: Posts and Telecom Press, 2013.08.

[6] Teng, Fei; Jia, Huaiyi, Implementation of Wireless Sensor Networks and WLAN Connectivity [J], Railway Computer Application, 2010.04.

[7] Zhang, Xiaowei, Adaptive TFRC Mechanism Based on Streaming Media Transmission in Wireless Networks [J], Zhejiang: Zhejiang Industrial University, 2012.

[8] Li, Siwei; Wang, Gui, etc., Wireless Network Optimization in Mobile Communication [M], Beijing: T singhua University Press, 2014.11.

[9] Xiang, Hao, IPv6 wireless sensor networks based on 6LoWPAN [D], Beijing: Beijing Science and Technology University, 2010.02.

[10] Li, Yinling, Network Engineering Planning and Design [M], Beijing: Posts and Telecom Press, 2012.07.

[11] Lin, Ying; Zhang, Yan; Kang, Yan, Network Attack and Defense Technology [M], Beijing: Tsinghua University Press, 2015.01. 